\title{
ENETS Consensus Guidelines for the Standards of Care in Neuroendocrine Neoplasms
}

DOI:

$10.1159 / 000471880$

Document Version

Accepted author manuscript

Link to publication record in Manchester Research Explorer

\section{Citation for published version (APA):}

Antibes Consensus Conference participants (2017). ENETS Consensus Guidelines for the Standards of Care in Neuroendocrine Neoplasms: Systemic Therapy Biotherapy and Novel Targeted Agents. Neuroendocrinology. https://doi.org/10.1159/000471880

\section{Published in:}

Neuroendocrinology

\section{Citing this paper}

Please note that where the full-text provided on Manchester Research Explorer is the Author Accepted Manuscript or Proof version this may differ from the final Published version. If citing, it is advised that you check and use the publisher's definitive version.

\section{General rights}

Copyright and moral rights for the publications made accessible in the Research Explorer are retained by the authors and/or other copyright owners and it is a condition of accessing publications that users recognise and abide by the legal requirements associated with these rights.

\section{Takedown policy}

If you believe that this document breaches copyright please refer to the University of Manchester's Takedown Procedures [http://man.ac.uk/04Y6Bo] or contact uml.scholarlycommunications@manchester.ac.uk providing relevant details, so we can investigate your claim.

\section{OPEN ACCESS}




\section{ENETS Consensus Guidelines for the Standards of Care in}

\section{Neuroendocrine Neoplasms: Systemic Therapy - Biotherapy and}

\section{Novel Targeted Agents}

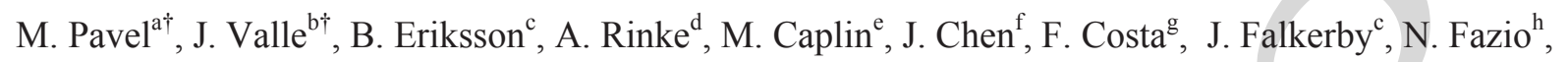
V. Gorbounova ${ }^{\mathrm{i}}$, W. de Herder ${ }^{\mathrm{j}}$, M. Kulke ${ }^{\mathrm{k}}$, C. Lombard-Bohas ${ }^{1}$, J. O’Connor ${ }^{\mathrm{m}}$, H. Sorbye ${ }^{\mathrm{n}}$, R. GarciaCarbonero $^{\circ}$ and all other Antibes Consensus Conference participants*

a. Department of Hepatology and Gastroenterology, Campus Virchow Klinikum, Charité Universitätsmedizin Berlin, Berlin, Germany

b. Department of Medical Oncology, The Christie NHS Foundation Trust, University of Manchester/Institute of Cancer Sciences, Manchester, United Kingdom

c. Department of Endocrine Oncology, Uppsala University Hospital, Uppsala, Sweden

d. Division of Gastroenterology and Endocrinology, University Hospital Marburg (UKGM), Marburg, Germany

e. Neuroendocrine Tumour Unit, Royal Free Hospital, London, United Kingdom

f. Department of Gastroenterology, The First Affiliated Hospital, Sun Yat-sen University, Guangzhou, China

g. Hospital Sirio Libanes, Sao Paulo, Brazil

h. Unit of Gastrointestinal Medical Oncology and Neuroendocrine Tumors, European Institute of Oncology, Milan, Italy

i. Department of Oncology, Institution of Russian Academy of Medical Sciences, Moscow, Russia

j. Department of Internal Medicine, Division of Endocrinology, ENETS Centre of Excellence Rotterdam, Erasmus MC, Rotterdam, the Netherlands

k. Dana-Farber Cancer Institute, Harvard Medical School, Boston, Massachusetts, USA

1. Medical Oncology Department, Hôpital Edouard Herriot, Hospices Civils de Lyon, Lyon, France

m. Department of Clinical Oncology, Institute Alexander Fleming, Buenos Aires, Argentina

n. Department of Oncology and Clinical Science, Haukeland University Hospital, Bergen, Norway

o. Medical Oncology Department, Hospital Universitario Doce de Octubre, Madrid, Spain

\section{${ }^{\dagger}$ shared first authors}

* For an alphabetical list of all other Antibes Consensus Conference participants, see Appendix. 


\title{
Corresponding author:
}

Marianne Pavel

Department of Hepatology, Gastroenterology \& Metabolic Diseases

Charité University Medicine

Campus Virchow Klinikum

Augustenburger Platz 1

13353 Berlin

Phone: +4930450553022

Fax: +4930450553902

Email: marianne.pavel@charite.de

\begin{abstract}
Systemic therapies established in the management of patients with NETs include somatostatin analogs and alpha-interferon, also referred to as biotherapy. Recent randomized controlled studies have extended the knowledge on the frequency of side effects associated with biotherapy. More recently, novel targeted drugs such as the mTOR inhibitor everolimus and the multiple tyrosine kinase Inhibitor sunitinib have been introduced in the management of NETs. Although targeted drugs are generally well tolerated, with most adverse events being of mild to moderate severity and manageable, novel targeted drugs exhibit a distinct adverse event profile that warrants guidance for appropriate diagnostic and therapeutic management. This is particularly important given the widespread and potentially longterm use of everolimus in a broad spectrum of NETs and of sunitinib in pancreatic NETs. This review will focus on the most relevant toxicities associated with biotherapy and novel targeted drugs and on their management. For each drug class indication, administration and dosing schedule, most frequent adverse events, actions and dose adjustments for adverse events as well as their monitoring are presented. This review further covers the evaluation of treatment effect, patient information, drug interactions, and information on pregnancy.
\end{abstract}




\section{Introduction}

Recently updated ENETS consensus guidelines provide a comprehensive overview on the use of systemic therapies and their indications in neuroendocrine tumors (NETs) [1] while standards of care (SOC) guidelines give advice on how to safely use drugs. Systemic therapies established in the management of patients with NETs include somatostatin analogs (SSA), alpha-interferon (IFN), also referred to as biotherapy, and novel targeted agents such as the mTOR inhibitor everolimus and the tyrosine kinase inhibitor (TKI) sunitinib. Other novel targeted agents evaluated in phase 2 trials (e.g. bevacizumab, axitinib and pazopanib) remain investigational (pending further validation in phase 3 studies and subsequent licensing) and will not be covered by these guidelines. SOC guidelines for systemic chemotherapy will be covered in a separate chapter [2].

Established systemic therapies are presented separately by drug class covering a brief summary of the indication, administration and dosing schedule, adverse events, actions and dose adjustments for adverse events, monitoring of adverse events, evaluation of treatment effect, patient information, drug interactions, and information on pregnancy and lactation. The severity of adverse events is indicated by Common Terminology Criteria for Adverse Events [3].

\section{SOMATOSTATIN ANALOGS}

\section{Indication}

Somatostatin analogs are indicated to treat symptoms related to peptide hypersecretion in functionallyactive NETs; this includes distinct clinical syndromes such as carcinoid syndrome, and syndromes related to duodenal or pancreatic NETs (e.g. vipoma, glucagonoma and gastrinoma) and, more rarely, PTH-related peptide-secreting tumors. Malignant somatostatin receptor-2 (sstr-2)-positive insulinoma may respond to a SSA, however it should be used with caution since hypoglycemia may worsen due to decreased secretion of glucagon [4].

Further, SSA are indicated to inhibit tumor growth in NET [5-6]. In this respect octreotide LAR is registered for midgut NET and NET of unknown primary, and lanreotide AG is registered for intestinal and pancreatic NET and NET of unknown primary. In general, somatostatin receptor status should be positive on somatostatin receptor imaging (SRI) if an SSA is going to be used with antiproliferative intent [7].

\section{Administration and Dosing Schedule}

Octreotide and lanreotide are registered drugs for NET in Europe. Octreotide is available as a shortacting subcutaneous (sc) formulation and as long-acting intramuscular injection (Octreotide LAR 10, 20 and $30 \mathrm{mg}$ ). Lanreotide, available only as long-acting formulations (30, 60, 90 and $120 \mathrm{mg}$ ), are administered by deep sc injection.

The optimal anti-proliferative doses have not been investigated; however, based on placebo-controlled 
phase 3 trials, octreotide LAR $30 \mathrm{mg}$ / month and lanreotide $120 \mathrm{mg}$ / month, respectively, are recommended. Although lower doses may be used for control of hormonal symptoms, knowledge of the anti-proliferative effect at the evaluated doses makes these doses preferable in these patients. The starting dose of octreotide LAR, according to the Summary of Product Characteristics (SPC) is 20 $\mathrm{mg}$ for symptom control (with 2-week overlap for patients established on sc octreotide) and $30 \mathrm{mg}$ as an anti-proliferative. Depending on the severity of symptoms and spread of the disease, some patients with functioning NET may need $30 \mathrm{mg}$ octreotide LAR. For lanreotide the SPC gives a starting dose range of 60-120 mg / month. Tolerability to a SSA may be tested with sc doses of octreotide (50-100 $\mu \mathrm{g}$ bid or tid, with escalation over 3-4 days, up to $600 \mu \mathrm{g} /$ day) in patients considered to be sensitive to side effects. In general, SSA treatment is maintained for as long as the patient is benefitting. In cases of refractory carcinoid syndrome or uncontrolled specific symptoms (e.g. diarrhea) related to functionally-active pancreatic NET (pNET), SSA doses may be increased to above-label doses by shortening the injection interval from 4-weekly to 3- or even 2- weekly if clinically required [8]. Alternatively, if an immediate effect is required, "rescue" octreotide 100-200 $\mu \mathrm{g}$ sc may be used on an as-required basis. In addition, if other drugs (e.g. IFN) or treatments (e.g. loco-regional therapies) are considered unfeasible, octreotide sc may be used on a regular schedule (e.g. 100-200 $\mu \mathrm{g}$ bid or tid). In patients with carcinoid syndrome, a continuous intravenous infusion of octreotide $(50-100 \mu \mathrm{g} / \mathrm{h})$ is required before surgery or any scheduled interventional therapies, continuing for $24-48 \mathrm{~h}$ depending on the type of surgery and extent of tumor burden. The infusion should start preferably 12 hours prior to surgery. For more details, see the chapter on perioperative management [9].

Above label dosages of SSA are not only used for improved syndrome control but also for tumor growth control in slowly growing tumors although not approved in this indication. Octreotide at a dose of $60 \mathrm{mg}$ ( 2 injections with $30 \mathrm{mg}$ every 4 weeks) has been explored in the NETTER-1 trial [10]; however more frequently $30 \mathrm{mg}$ is used every 3 or even every 2 weeks. Lanreotide $120 \mathrm{mg}$ every 2 weeks for antiproliferative purpose is under evaluation in a clinical trial (NCT02651987; www.clinicaltrials.gov). A higher than standard dose of either octreotide or lanreotide can be considered outside of clinical trials in selected cases, with radiological progression within a long time frame (2-3 yrs) or low tumor burden or any other setting where alternative treatment options such as everolimus or PRRT in intestinal NET, and chemotherapy or targeted agents in pNET seem not appropriate or feasible.

\section{Adverse events}

Somatostatin analogs are generally well tolerated [11]; in the phase 3 trials, discontinuation due to treatment-related adverse events (AEs) was reported in only $1 \%$ of patients with entero-pancreatic NETs with lanreotide [6] and 12\% of patients with midgut-NETs with octreotide LAR [5].

The most frequent AEs are GI disorders including abdominal discomfort or pain, nausea, flatulence, 
and diarrhea. In general, these AEs resolve within 2-3 weeks on treatment. To reduce GI AEs doseescalation of SSA may be instituted as described previously. Transient hypoglycemia, usually not clinically significant, may occur and can be avoided by regular food intake. Bradycardia, a very rare $\mathrm{AE}$, has been reported after sc injection of octreotide or when given as an intravenous (iv) bolus in larger doses [12-15] it should be considered if other causes have been ruled out.

Somatostatin analogs modestly suppress thyroid stimulating hormone (TSH) secretion, but in general do not cause clinically-evident hypothyroidism requiring thyroid hormone replacement [16].

Adverse events occurring with long-term use of SSAs include diarrhea/ steatorrhea due to pancreatic enzyme insufficiency; sludge in the gallbladder or gallstones; hyperglycemia, hypocalcaemia, and vitamin B12 deficiency. Deficiency of one or more fat soluble vitamins has been described in more than $70 \%$ and multiple deficiencies in $38 \%$ of patients with long-term SSA use for $\geq 18$ months. These included deficiencies for vitamin A, D, E, K and E in erythrocytes in $6 \%, 28 \%, 15 \%, 63 \%$ and $58 \%$ of the patients [17]. Subcutaneous nodules at the site of depot injections are not uncommon. Hair loss is reported in few patients.

\section{Actions and dose adjustments of SSA for adverse events}

In general dose reductions, to 10-20 mg octreotide LAR/ month or 60-90 mg lanreotide AG / month for AEs, are rarely needed.

Pancreatic enzyme insufficiency (PEI) is a common AE during treatment with SSAs [18]; if diarrhea / lose stools occur, determination of fecal elastase to establish the diagnosis of PEI is recommended and substitution with pancreatic enzymes, if confirmed. Other causes of diarrhea related to surgery (e.g. bacterial overgrowth, short bowel syndrome, bile acid loss) or more general causes of diarrhea (e.g. lactose intolerance) should be considered and excluded. Use of cholestyramine in cases of bile acid loss or loperamide, if no specific reason for diarrhea can be identified, is recommended.

Gallstones have been reported in $10 \%$ of the patients with lanreotide AG and $14 \%$ with octreotide LAR in the CLARINET and PROMID trials, respectively [5, 6]. If symptomatic, the gallbladder should be removed. In cases of planned surgery for primary tumor or metastases, or abdominal surgery unrelated to NET, a prophylactic cholecystectomy can be considered.

Hyperglycemia occurred in 5\% of patients with $120 \mathrm{mg}$ lanreotide AG / month in the CLARINET study, and was reported in $5 \%$ of patients with carcinoid syndrome when treated with $40 \mathrm{mg} / \mathrm{month}$ octreotide LAR (2\% of grade 3-4) [19]. Patients with impaired glucose tolerance or diabetes mellitus should be monitored closely when SSA therapy is initiated; initiation or adjustment of antidiabetic therapy might be necessary.

Vitamin B12 deficiency may occur with SSA therapy [20] and should be substituted. Similarly, in cases of Vitamin D deficiency or deficiency of other fat soluble vitamins substitution is recommended. Thyroid hormone replacement is indicated with the occurrence of hypothyroidism.

In $\leq 1 \%$ of patients, severe and/or durable SSA-related diarrhea; or increased diarrhea and/or flushing 
in carcinoid syndrome due to paradoxical release of mediators; or exacerbation of hypoglycemia in metastatic insulinoma may occur [21]. In such cases, SSA should be stopped and an alternative treatment considered.

\section{Monitoring of adverse events}

Before starting a SSA the following investigations should be performed:

- Physical examination with determination of blood pressure and heart rate

- Laboratory: blood cell count, transaminases, bilirubin, blood glucose, HbAlc, electrolytes (including calcium), creatinine, TSH, vitamin D and vitamin B12

- Electrocardiogram (ECG)

- Ultrasound of the gallbladder

During follow up, the following investigations are advised:

- Physical examination with determination of blood pressure and heart rate

- Laboratory: blood cell count, transaminases, bilirubin, blood glucose, HbA1c (if hyperglycemia develops), electrolytes (including calcium), creatinine at 1 month and 3monthly thereafter; vitamin B12, fat-soluble vitamins and TSH should be monitored annually

- Fecal elastase if steatorrhea occurs

- ECG if clinically indicated (bradycardia, signs of chronic heart failure); in patients with carcinoid heart disease ECG and echocardiography should be regularly performed (q 3-12 mo depending on the severity)

- Ultrasound of the gallbladder if clinically indicated

\section{Evaluation of treatment effect}

\section{Biochemical control:}

Before starting therapy the following biomarkers should be measured:

- Chromogranin A (CgA); Neuron-specific enolase (NSE) may be considered in G2 NET if CgA is normal.

- 5-hydroxy-indole acetic acid (5-HIAA) in blood or 24-h urine (collection on acetic acid or hydrochloric acid, depending on local laboratory requirements) in patients with (or suspected) carcinoid syndrome.

- Additional tumor markers, if indicated, depending on type of tumor (e.g. gastrin, vasoactive intestinal polypeptide (VIP), glucagon, insulin).

At follow-up biomarkers elevated at baseline should be re-measured at 3 months; then in parallel to imaging (see below); if specific symptoms increase or new symptoms appear; or clinical suspicion of progressive disease. 


\section{Tumor response:}

Before treatment and during follow-up the following investigations are recommended:

- Cross-sectional imaging, either CT or MRI of the liver and abdomen every 3-6 months depending on tumor grade and primary tumor origin (e.g. 6-monthly in grade 1 (G1) small intestinal NET, and every 3 months in G2 pNET); the interval can be prolonged to one year if stable disease after 3-5 years.

- Somatostatin receptor (SR) imaging (if not previously available or older than 1 year); either an octreoscan or ${ }^{68} \mathrm{Ga}-\mathrm{SR}-\mathrm{PET} / \mathrm{CT}$ to assess somatostatin receptor status and for whole body staging [7]. Repeat SR-Imaging after 12-24 months according to ENETS guidelines [22].

- CT of the thorax if SR imaging revealed thoracic lesions; then every 3-12 months depending on tumor grade and growth rate.

\section{Patient information}

- Patients with carcinoid syndrome should receive information on dietary restrictions (to avoid food that elevates serotonin in blood) before biochemical assessment of 5-HIAA in blood or 24-h urine. This is particularly important if the diagnosis of carcinoid syndrome needs to be established in patients with mild symptoms suspicious of carcinoid syndrome. Drugs that can interfere with 5HIAA determination and are not necessarily needed should be avoided.

- Certain foods (with a high or moderate amount of amines; very spicy foods; or high-fat meals) and alcoholic beverages may induce flushing and/or diarrhea, and thus should be avoided.

\section{Drug interactions}

- The bioavailability of bromocriptine, quinidine, or terfenadine may be increased by octreotide or lanreotide.

- Cyclosporin: reduction of serum cyclosporine levels have been recorded that may increase the risk of transplant rejection.

- Patients receiving insulin, oral antidiabetic agents, beta blockers (e.g. propranolol, bisoprolol, carvedilol, atenolol), calcium channel blockers, or agents to control fluid and electrolyte balance, may require dose adjustments of these treatments whilst on SSA.

Precise information on drug interactions can be retrieved from the U.S. Food and Drug Administration (FDA) [23].

\section{Pregnancy and lactation}

Data concerning the use of SSA in pregnancy are very limited. Pregnancy has been reported in women while on octreotide or lanreotide; based on these case reports and animal studies, SSA seem not to be teratogenic or genotoxic $[24,25]$. However, octreotide does cross the placenta, and data are too limited to fully assess the human fetal risk [25]. Therefore, the use of SSA should only be considered if the 
assumed benefits far outweigh potential risks following multidisciplinary discussion between the oncologist, endocrinologist and gynecologist.

There are no data on the excretion of SSA into human milk, but animal studies have shown excretion of octreotide in breast milk, so it is preferable that patients do not breast-feed while on SSA treatment or this should be done with great caution [25].

\section{INTERFERON}

\section{Indication}

Interferon (IFN)-alpha-2b is registered in Europe for the treatment of NETs associated with carcinoid syndrome; it is also used for functionally-active pNETs (e.g. vipoma, glucagonoma, insulinoma) to improve symptoms related to hypersecretion of amines and peptides. In general, it is used as an add-on therapy to SSA in refractory carcinoid syndrome or if SSAs are not the preferred choice (e.g. negative SSTR status) or not tolerated. Uncontrolled and prospective randomized trials have shown activity of IFN similar to that of SSA in gastro-entero-pancreatic NETs [26-28]. A recent large controlled trial in advanced "carcinoids" supported its anti-proliferative activity [29]. However, IFN is not registered as an anti-proliferative, but may be considered as an option, particularly in patients with non-pancreatic NET [1].

\section{Administration and Dosing Schedule}

The most frequently used recombinant IFN preparations have been IFN alpha-2b and IFN- alpha-2a, which differ from each other by a single amino acid residue. More recently, pegylated (PEG) forms of IFN are in clinical use. Standard IFN-alpha is administered sc thrice-weekly; pegylated IFN-alpha once-weekly. According to the SPCs (http://www.ema.europa.eu/ema/) and ENETS Guidelines, the standard dose of IFN alpha-2b (IntronA ${ }^{\circledR}$ ) is 3-5 MU three times a week [1]. A starting dose of $3 \mathrm{MU}$ three times a week is recommended with titration of the dose according to tolerability. Standard dose of IFN-alpha-2a (Roferon $\left.{ }^{\circledR}\right)$ is 3-4.5 MU three times a week. For safety reasons the white blood cell count should not be lower than $3 \times 10^{9} / 1$.

PEG-IFN alfa-2b (PEG-Intron ${ }^{\mathrm{TM}}$ ), at a dose of $0.5 \mu \mathrm{g} / \mathrm{kg}$, has been used in NETs [30, 31]; starting at $50 \mu \mathrm{g} /$ week sc the dose is increased in 4-weekly intervals up to150 $\mu \mathrm{g} /$ week (depending on weight and tolerability). Dose increase to150 ug/week is very rarely necessary.

IFN-alpha should be interrupted 3-4 weeks before surgery, PRRT or TAE/ TACE and for at least 2-4 weeks thereafter (in exceptional cases it may not be feasible to interrupt IFN due to severe carcinoid syndrome, especially in patients with high tumor burden). 


\section{Contraindication}

IFN should not be used in patients with severe autoimmune diseases (e.g. rheumatoid arthritis and systemic lupus erythematosus (SLE)); if psychiatric disorders (e.g. depression or psychosis) are present or previously reported; in patients with severe renal or hepatic insufficiency or epilepsy; or after organ transplantation.

Caution is advised in elderly patients ( $>70$ years) because of the frequency of impaired hepatic, renal, bone marrow, or cardiac function; concomitant diseases; or other drug therapy.

\section{Adverse events}

In comparison to SSA, IFN needs to be interrupted more frequently for AEs [26-28]; these include:

- Fever and flu-like symptoms (chills, malaise, headache, myalgia, tachycardia) are common (affecting approx. 90\% of patients) during therapy with standard IFN-alpha; occurring mostly within $1-2 \mathrm{~h}$ after injection.

- Fatigue or weight loss occurs in about 50\% of patients, (26\% of grade 3-4) [28].

- Myelosuppression leading to dose-dependent leucopenia (7\% of grade 3-4), lymphopenia, anemia $(30 \%)$, and thrombocytopenia (20\%).

- Autoimmune disorders occur in up to $20 \%$ of patients, most frequently hyperthyroidism (Basedow disease) or Hashimoto's disease; others include vasculitis, Raynaud's phenomenon, rheumatoid arthritis and SLE.

- Psychiatric side effects, especially depression.

- Reversible hair loss.

- Polyneuropathy (rarely).

- Mild hepatotoxicity (elevation of transaminases) in 30\% of patients; fatal hepatotoxicity has been reported, but is extremely rare.

- Elevated triglyceride levels.

Adverse events of grade 3-4 are less frequently reported with PEG-IFN-alpha $2 \mathrm{~b}$ (fatigue $25 \%$, myalgia $<10 \%$, headache $0 \%$ ) [30], and PEG-IFN-alpha 2a [31].

\section{Actions and dose adjustments of IFN for adverse events}

If standard IFN therapy is not tolerated PEG-IFN may be used instead unless serious AEs occurred, such as psychiatric disorders including depression, or serious autoimmune diseases.

- Flu-like symptoms: may be attenuated by pre-medication with paracetamol (1000 mg orally or rectally) or aspirin/ NSAIDs.

- $\quad$ For grade 2-3 fatigue or weight loss, reduce dose or stop treatment.

- $\quad$ Reduce IFN dose if leucocytes drop below $3 \times 10^{9} / 1$.

- Autoimmune hyperthyroidism may be transient but may also require thyrostatic drugs (e.g. a 
thioamide). If tachycardia is present, beta-blockers may be used. Hashimoto disease requires thyroid hormone substitution.

Discontinuation of IFN is required under the following conditions:

- Psychiatric disorders

- Severe bone marrow depression

- Severe weight loss (grade 3)

- Severe hepatic disease; discontinue IFN for any patient developing signs or symptoms of liver failure.

- Autoimmune disease such as SLE, rheumatoid arthritis

\section{Monitoring of adverse events}

Patients should be seen regularly by a physician every 4-6 weeks initially for assessment of the general condition, performance status (PS) and body weight.

Before starting IFN the following investigations should be performed

- Laboratory: blood cell count, transaminases, bilirubin, albumin, prothrombin time, blood glucose, electrolytes including calcium, creatinine, thyroid function tests (according to local practice), triglycerides.

- Electrocardiogram (ECG).

In the follow up the following investigations are recommended:

- Laboratory: blood cell count, transaminases, bilirubin, blood glucose, electrolytes including calcium, creatinine, triglycerides at 1 month and 3-monthly thereafter; vitamin B12 and TSH once a year.

- ECG if clinically indicated (tachycardia, arrhythmia).

\section{Evaluation of treatment effect}

\section{Biochemical control:}

Before starting therapy the following biomarkers should be measured:

- $\mathrm{CgA}$; NSE may be considered in G2 NET.

- 5-HIAA in blood or 24-h urine (collection on acetic acid or hydrochloric acid, depending on local laboratory requirements) in patients with (or suspected) carcinoid syndrome.

- Additional tumor markers, if indicated, depending on type of tumor (e.g. gastrin, VIP, glucagon, insulin). 
At follow-up biomarkers elevated at baseline should be re-measured at 3 months; then in parallel to imaging (see below); if specific symptoms increase or new symptoms occur; or clinical suspicion of progressive disease.

\section{Tumor response:}

Before treatment and during follow-up the following investigations are recommended:

- Cross-sectional imaging, either CT or MRI of the liver and abdomen every 3-6 months depending on tumor grade and primary tumor origin (e.g. 6-monthly in G1 small intestinal NET, and every 3 months in G2 pNET); the interval can be prolonged to one year if stable disease after 3-5 years.

- CT of the thorax if SR imaging revealed thoracic lesions; then every 3-12 months depending on tumor grade and growth rate.

\section{Patient information}

- Patients should be informed that the most common AE is flu-like symptoms (fever, headache, fatigue, anorexia, and nausea or vomiting); these usually decrease in severity as treatment continues; symptoms may be minimized by bedtime doses and premedication with paracetamol.

- In case of suspected infections patients should be advised to see their physician to determine white blood cell count and infective markers (c-reactive protein/pro-calcitonin).

- Patients should be informed that IFN may cause drowsiness or dizziness and that depressed mood may occur, even after long-term use, and requires prompt consultation.

- Patients are to be advised that they may develop fatigue, somnolence, or confusion during treatment with interferon, and therefore it is recommended that they avoid driving or operating machinery

- IFN should be stopped 3-4 weeks prior to surgery and after surgery depending on co-morbidities/ complications to decrease perioperative risk of infections.

\section{Drug interactions}

There are multiple drug interactions with IFN-alpha $2 \mathrm{~b}$ and IFN-alpha-2a; please refer to the product's SPC. Selected drug interactions include:

- Inhibition by IFN of the hepatic enzymes CYP1A2 and CYP2C19.

- Ribavirin: concurrent treatment may increase the risk of hemolytic anemia.

- Theophylline: IFN may inhibit hepatic metabolism of theophylline, therefore monitor for increased levels / effects.

- Zidovudine: IFNs may decrease zidovudine metabolism; the neutropenic effects of zidovudine and IFN may be synergistic and require monitoring.

Precise information on drug interactions can be retrieved from the U.S. Food and Drug Administration 
(FDA) [23].

\section{Pregnancy and lactation}

Contraceptive measures are recommended during therapy. Pregnancies have been described with IFN therapy [32], although no congenital malformations have been reported in a series of 27 infants, intrauterine growth retardation was present in $22 \%$.

Lactation: effect is undetermined; discontinue breast-feeding or the drug.

\section{TARGETED THERAPY}

This section focuses on novel targeted drugs, everolimus and sunitinib, which are registered for use in advanced NETs; therapy should be in the hands of experienced clinicians. In fact, several physicians may be involved in the management of AEs, including the primary physicians. Awareness of potential side effects and instruction of the patient may help to optimize therapy management. The aim of these SOC guidelines is to provide guidance for diagnosis and management of AEs.

\section{Everolimus}

\section{Indication}

Everolimus is an inhibitor of the mammalian target of rapamycin (mTOR), an intracellular protein kinase downstream of the phosphatidylinositol 3-kinase/AKT pathway involved in key components of tumorigenesis, including cell growth, proliferation, and angiogenesis.

Everolimus is registered for therapy of advanced, progressive pNETs and for advanced, progressive G1/G2 non-functional NETs of gastrointestinal or lung origin [33, 34]. Everolimus may improve symptoms from NET-related endocrine hypersecretion; particularly in patients with metastatic insulinomas [35].

\section{Administration and Dosing Schedule}

Everolimus recommended dose is $10 \mathrm{mg}$ /day as a continuous oral treatment. Prescribers should refer to the SPC from the European Medicines Agency (EMA) for updated prescribing information (http://www.ema.europa.eu/ema/). Side effects may lead to treatment discontinuation in $17-25 \%$ of patients and to dose reductions in $60 \%$ of patients to $5 \mathrm{mg} /$ day or even $5 \mathrm{mg}$ every other day. Treatment interruption is advised if grade 3-4 treatment-related AEs develop, until recovery to grade $<1$. Then treatment may be reinitiated generally with dose adjustments as indicated below. Hepatic impairment will increase the exposure to everolimus and requires dose reduction to $7.5 \mathrm{mg}$ daily in patients with mild hepatic impairment (Child-Pugh class A) and $5 \mathrm{mg}$ daily in patients with moderate hepatic impairment (Child-Pugh class B). The dose may be further decreased by one dose level if not well tolerated. 
Interruption of everolimus should be considered at least 2 weeks, preferably 3-4 weeks prior to surgical interventions to minimize potential immunosuppressive effects and increased infection risk.

\section{Adverse events}

Although generally well tolerated, with most adverse events of mild to moderate severity and manageable, everolimus exhibits a distinct AE profile. Frequencies of AEs are extracted from RADIANT-2 and RADIANT-3 trial data $[33,36]$; the most relevant toxicities include:

- Stomatitis: oral ulceration (inflammation of the mucous membranes of the oral cavity, inner surface of the lips or tongue) represents the most common AE reported in $>60 \%$ of patients $(7-9 \%$ of grade 3-4). It usually occurs within the first 8 weeks of treatment, with decreasing prevalence thereafter; mucosal lesions usually resolve within 10-14 days of treatment discontinuation.

- Skin rash (featuring papulopustular or maculopapular eruptions which may be pruritic) is a common AE; reported in $29-49 \%$ of patients, it is mostly mild to moderate in severity. Impaired wound healing may occur.

- Diarrhea occurs in 30\% of patients and is mostly mild to moderate (grade 3-4 in 3-7\% of patients).

- Myelotoxicity: anemia or thrombocytopenia occurs in less than $20 \%$ of the patients, and is rarely $(\sim 5 \%)$ grade $3-4$.

- Infections occur in around $20 \%$ of the patients and may range from a simple cold to pneumonia or opportunistic infections including invasive fungal infections (e.g. aspergillosis or candidiasis). Although most infections in the trials were mild some were severe (2-7\%) and culminated in respiratory failure and sepsis and were occasionally fatal [37]. There is a risk of reactivation of latent hepatitis B virus infection.

- $\quad$ Non-infectious pneumonitis occurs in $12-17 \%$ of patients and is characterized by non-infectious lung infiltrates and negative blood and bronchoalveolar lavage bacterial tests. Typical findings are ground-glass attenuation and focal consolidation, mainly in the lower lobes on CT or chest X-ray. Clinical symptoms such as dyspnea or cough are typical, but may be absent.

- Metabolic abnormalities include hyperglycemia, hyperlipidemia and hypophosphataemia. Hyperglycemia of any grade was reported in $12 \%$ of patients with everolimus and octreotide LAR in RADIANT-2, and was in a similar range (13\%) in pNET in RADIANT-3; grade 3-4 hyperglycemia occurred in 5\%. Raised triglyceride and cholesterol levels were reported in 39 and $66 \%$, respectively. Hypophosphatemia (which may cause muscle weakness) occurred in $40 \%$ of pNET patients, $10 \%$ of which were grade 3-4.

- Renal function: mild reversible creatinine elevation has been reported in around $20 \%$ of patients with pNET (RADIANT-3); 1\% were graded 3-4.

- Asthenia or fatigue is reported in one third of treated patients, although it is generally mild to moderate (only in $1-2 \%$ it is of grade $3-4$ ). 
- Peripheral oedema occur in $13-20 \%$ of the patients, but are very rarely severe ( $\leq 1 \%$ grade $3-4)$.

\section{Actions and dose adjustments of everolimus for adverse events [38]}

o Stomatitis is mostly of grades 1-2; early intervention to prevent worsening is important. Spicy and salty foods should be avoided. Regular preventive oral rinses with isotonic saline, or alcohol- and peroxide-free mouthwash or sage tea are recommended. The use of dexamethasone mouth rinse may be considered to prevent stomatitis in potentially susceptible patients, based on the findings of the SWISH trial in postmenopausal women with breast cancer receiving everolimus and exemestane. The study demonstrated a lower incidence of stomatitis ( $2.4 \% \geq$ grade 2 stomatitis at 8 weeks), compared with $33 \%$ in a historical control) when concomittant dexamethasone mouth rinse was used [39].

o Grade 1 (minimal symptoms; normal diet); continue everolimus, use oral mouth wash solutions.

o Grade 2 (symptomatic, but patient is able to eat adequately); continue therapy if possible, or interrupt, until Grade $\leq 1$; use topical therapies including corticosteroids.

o Grade 3 (insufficient oral fluid and food intake): interrupt therapy until recovered to grade $\leq 1$, thereafter continue with $5 \mathrm{mg}$ daily.

o Grade 4 (parenteral nutrition required): terminate everolimus.

- Skin rash; in general, skin lesions resolve spontaneously during treatment.

o Grades 1-2 localized skin rash can be treated with topical cortisone creams and moisturizers.

o For Grades 3-4 skin lesions and generalized rash, interruption of everolimus and low-dose corticosteroids (e.g., prednisone 10-25 mg/day orally) can be considered until grade $\leq 1$.

- Non-infectious pneumonitis

o Grade 1 (asymptomatic radiographic findings only); observation, low dose CT of the chest after 4-6 weeks recommended

- Grade 2 (symptomatic, but no impairment of daily activity); interrupt everolimus until grade $\leq 1$, then re-initiate treatment at $5 \mathrm{mg}$ daily.

o Grade 3 (impairment of daily activity; oxygen required); interrupt everolimus until recovery to grade $\leq 1$; then resume at one dose level lower, if grade 3 pneumonitis recurs after re-exposure at a lower dose (e.g. $5 \mathrm{mg} /$ day), consider discontinuation of everolimus

o Grade 4 (mechanical ventilation required, life-threatening); terminate everolimus

- Infections

o For all infections of grades 2-3 prompt treatment with appropriate antibiotic, antifungal, or antiviral is required, with interruption of everolimus therapy. After recovery re-exposure at a lower dose is feasible. If grade 4 infections occur, everolimus should be discontinued.

- Hyperglycemia/ diabetes (hyperglycemia grade $\geq 2$ ) should be treated according to EASD and 
ADA guidelines:

o Grades 1-2: blood glucose $<250 \mathrm{mg} / \mathrm{dl}$; continue everolimus, start antidiabetic drugs if glucose $>160 \mathrm{mg} / \mathrm{dl}$ (grade 2); monitoring of glucose by the patient.

o Grade 3: Blood glucose $>250-500 \mathrm{mg} / \mathrm{dl}$; interrupt everolimus, until hyperglycemia resolves, and then restart therapy generally at reduced doses $(5 \mathrm{mg} / \mathrm{d})$, although dose titration up to $10 \mathrm{mg} / \mathrm{d}$ may be considered if hyperglycemia is adequately controlled.

o Grade 4: Blood glucose $>500 \mathrm{mg} / \mathrm{dl}$, discontinue everolimus

- Hyperlipidemia, hypertriglyceridemia and hypercholesterolemia should be treated according to standard guidelines [40]:

o If triglyceride levels are $\geq 500 \mathrm{mg} / \mathrm{dl}$ use fibrates

o If statins are used, be aware of potential interactions of statins with CYP3A4 enzymes

- Hypophosphatemia; weekly to monthly monitoring of serum phosphate depending on the severity.

o Grades 1-2: should be managed with diet and oral phosphate (1000-2000 mg/ day divided in 4 doses) depending on the severity of phosphate depletion.

o Grades 3 and 4: severe hypophosphatemia $(<2 \mathrm{mg} / \mathrm{dl}$ and $<1 \mathrm{mg} / \mathrm{dl}$, respectively) can lead to respiratory and heart failure; and requires hospitalization for iv phosphate therapy. Everolimus therapy should be interrupted.

- Creatinine elevation

o Grades 1-2: avoid nephrotoxic drugs; adjust anti-hypertensive medication Grades

o 3-4: interrupt everolimus; after normalization of creatinine in grade 3 events everolimus can be re-started at a lower dose.

In general, discontinuation of therapy is recommended for grade 4 adverse events according to CTC [3], but there may be few exceptions, depending on the circumstance, in which clinical judgement can be used and it might be considered to restart therapy with dose reduction.

\section{Monitoring of adverse events}

Patients should be reviewed every 4 weeks while on treatment; with long-term therapy ( $>2 \mathrm{yr})$ and good tolerability clinical follow-up can be reduced to 3-monthly.

Before starting everolimus the following investigations should be done:

Thorough physical investigation and assessment of comorbidities (e.g. diabetes, cardiovascular or lung disease, infections).

- Pre-existing infections should be treated appropriately and should have fully resolved before starting treatment with everolimus.

- Laboratory: Blood cell count, blood glucose, electrolytes, calcium, serum phosphate, creatinine, transaminases, cholesterol and triglycerides (in general, lipid and triglyceride levels should be 
normalized before initiating everolimus therapy).

- Antibody status for HBV and HCV should be determined. In case of positive serology, PCR for HCV RNA and HBV DNA, respectively, should be determined.

- If clinically indicated, rule out tuberculosis by IFN $\gamma$ test (e.g Quantiferon ${ }^{\circledR}$ ) or Mantoux reaction test.

- A baseline a (low dose) CT scan may be considered optionally for reference purposes of a later possible pneumonitis.

During treatment the following investigations are recommended:

- Assessment of the general condition, PS and weight every 4 weeks.

- Laboratory parameters should be measured regularly after 2 weeks, and thereafter at least on a monthly basis (more frequently if needed) for 3 months: blood cell count, blood glucose, electrolytes, calcium, serum phosphate, creatinine, cholesterol and triglycerides. With long-term treatment, particularly if no abnormal findings, laboratory evaluations may be prolonged to 2-3 months intervals.

- For patients with a history of HCV, routine HCV RNA monitoring is mandatory; and HBV DNA for history of hepatitis B.

- Physicians should be vigilant for symptoms and signs of infection; if a diagnosis of infection is made, initiate appropriate treatment promptly and consider interruption or discontinuation of everolimus.

\section{Evaluation of treatment effect}

Biochemical markers: CgA or NSE (if elevated prior to therapy); specific biomarkers (depending on functionality).

Tumor follow-up: Conventional imaging, either CT or MRI (whatever method depicts tumor lesions most accurately) every 3 months. With long-term therapy and stable disease the staging interval may be prolonged to 6-monthly. Although RECIST evaluation has limitations in NET response assessment, these criteria are still considered the reference method [41].

\section{Patient information}

The patient should be advised

- that stomatitis is a frequent, but mostly transient and mild event.

- that infections may occur with everolimus therapy, and that oral corticosteroids should not be given while treatment with everolimus is ongoing.

- that live vaccines need to be avoided.

- that an interruption of everolimus for at least 2 weeks, preferably 3-4 weeks prior to scheduled surgery or any other intervention as well as 2 weeks thereafter is needed.

- $\quad$ not to drink grapefruit juice due to its impact on metabolism of everolimus. 
- that contraception in females and males is required during therapy.

\section{Drug interactions}

Everolimus is a substrate of cytochrome P450 (CYP3A4), and interacts with p-glycoprotein (pGP); thus a number of drugs may change the blood level of everolimus. Inhibitors of CYP3A4 and pGP that are commonly in use include ketoconazole, erythromycin, verapamil, diltiazem, cimetidine, fluconazole, and cyclosporine. Antifungal systemic therapy may increase serum concentrations of everolimus and everolimus should be adjusted (or serum levels monitored closely for elevation). Before starting everolimus careful consideration of concomitant medication is needed to rule out potential interactions [23].

\section{Pregnancy and lactation}

Pregnancies whilst on everolimus-based immunosuppression in kidney transplant patients have been reported [42]. In animal studies toxic effects have been seen; men and women should adhere to contraceptive methods and should not perform breastfeeding while taking everolimus.

\section{Sunitinib}

\section{Indication}

Sunitinib malate is an oral multi-targeted tyrosine kinase inhibitor of vascular endothelial growth factor receptors (VEGFRs), platelet-derived growth factor receptors (PDGFRs), KIT, and RET. It is licensed for patients with progressive, unresectable, locally advanced or metastatic, well-differentiated pNET based on a placebo-controlled trial [43]; trials are ongoing to evaluate the efficacy of sunitinib in non-pancreatic NET.

\section{Administration and Dosing Schedule}

The recommended dosing regimen of sunitinib is $37.5 \mathrm{mg}$ orally once daily as continuous therapy; note that this dosing schedule differs from that used for gastrointestinal stromal tumors and advanced renal cell cancer ( $50 \mathrm{mg}$ daily for 4 weeks followed by 2 weeks off therapy (" $4 / 2$ schedule")). Prescribers should refer to the SPC for updated prescribing information (http://www.ema.europa.eu/ema/). The dose may be reduced to $25 \mathrm{mg} /$ day or $12.5 \mathrm{mg} /$ day depending on tolerability.

Due to the mechanism of action sunitinib should be interrupted for 3-4 weeks prior to, and 2 weeks after surgical interventions due to potential bleeding risk and impact on wound-healing. 


\section{Adverse events}

The most frequent AEs reported in the phase 3 pNET trial were diarrhea, nausea, asthenia, vomiting, and fatigue; these were mainly grade 1-2. The commonest grade 3-4 toxicities were neutropenia, hypertension, diarrhea, asthenia and fatigue. Discontinuation due to AEs occurred in 15\% of patients; and $31 \%$ of patients required at least one dose reduction [43, 44].

- Hypertension of any grade occurred in 26\% of patients (grade 3-4 in 10\%); it is an on-target AE associated with VEGF-1 inhibition.

- Neutropenia occurred in $29 \%$ of patients overall; only $12 \%$ were of grade 3-4 and there were no reported cases of febrile neutropenia in the phase 3 study. It is usually short-lived and may be due to neutrophil margination (vs. true neutropenia) as neutrophil levels have been reported as returning to normal within $24 \mathrm{~h}$ of corticosteroid administration.

- GI toxicity; the commonest AE was diarrhea (59\%) although only 5\% were of grade 3-4. Typical onset is approx. 3 weeks into treatment and rarely starts beyond 6 months. Other causes (pancreatic duct obstruction or SSA-induced) need to be considered. Dose modification of sunitinib is rarely necessary. Nausea (occurring in $45 \%$ of patients) and vomiting (34\%) were generally mild or moderate in severity and grade 3-4 events were rare; other causes (such as duodenal obstruction, electrolyte imbalance, etc.) should be considered and treated. Dysgeusia may occur.

- Fatigue and asthenia occur in about $1 / 3$ of patients (but grade 3-4 in only $5 \%$ ). Fatigue usually develops during the first month of treatment, with the highest incidence frequently noted after 2-3 months. Other causes (such as hypothyroidism, anemia, malignancy itself, depression, electrolyte imbalance and dehydration) need to be considered and treated.

- Dermatologic AEs occurred frequently in the phase 3 pNET trial including hair and skin depigmentation (29\% of patients), hand-foot syndrome (HFS; $23 \%)$, rash (18\%) and dry skin (15\%). Grade 3-4 HFS ( $6 \%$ of patients) is painful and may be debilitating; it particularly affects highpressure ("wear and tear") areas of the hands and feet [45].

- Oral toxicity, stomatitis, and mucositis: although this is frequently reported (48\%) only $6 \%$ are severe (grade 3-4); it often occurs during the first month of treatment (peak severity occurs during the second and third months) and appears to stem from functional irritation of the mucosa [46].

- Thyroid dysfunction; hypothyroidism occurred in 7\% of all cases, all of grade 1-2 severity; the exact mechanisms are not known. Longer duration of sunitinib treatment appears to increase the incidence of all-grade hypothyroidism [47].

- Cardiovascular events; according to the SPC, sunitinib may prolong the QT-interval in a dosedependent manner, which may lead to an increased risk of ventricular arrhythmias including torsade de pointes (observed in $<0.1 \%$ of sunitinib-exposed patients). Sunitinib should be used with caution in patients with a history of QT-interval prolongation, those taking anti-arrhythmics, or those with relevant pre-existing cardiac disease, bradycardia, or electrolyte disturbances. Note 
that patients with QTc intervals of $>450 \mathrm{msec}$ for males or $>470 \mathrm{msec}$ for females were excluded from the pivotal study.

- Thromboembolic events were not reported in patients treated with sunitinib in the phase 3 pNET trial.

\section{Dose adjustments for adverse events}

- Hypertension; this is usually manageable and allows continued sunitinib therapy:

o Grades 1-2 (BP elevated but $<160 /<100$ ): use vasodilatory antihypertensive drugs (e.g. ACE inhibitors or angiotensin II receptor antagonists in preference to calcium channel blockers, diuretics or beta-blockers. Avoid CYP4503A4 inhibitors such as diltiazem and verapamil (calcium channel blockers); this is not an issue with the dihydropyridine class of calcium channel blockers. Use caution with beta-blockers and calcium channel blockers that cause PR elongation.

o Grade 3 ( $\mathrm{BP} \geq 160 / 100)$ : interrupt sunitinib until hypertension is under control, resume at same dose initially but may need to reduce dose if grade 3 hypertension recurs.

o Grade 4 (life-threatening, e.g. malignant hypertension, neurological deficit, hypertensive crisis): discontinue sunitinib.

- Neutropenia; no dose adjustments are required for grades 1-2 neutropenia.

o Interruption of sunitinib is recommended for grades 3-4 events; in case of grade 3 events reintroduction of sunitinib at a reduced dose once neutropenia has improved to grade $\leq 2$.

o In grade 4 events discontinuation of therapy is recommended, however, if lasting $<1$ week and without any clinical symptoms/ sequelae reintroduction of sunitinib at a lower dose can be considered in selected cases.

o For prolonged neutropenia, the use of growth factors may be considered in line with NCCN guidelines [48].

o GI toxicity:

o Diarrhea: oral hydration and anti-diarrheals usually suffice for grades 1-2; interruption of sunitinib is recommended for grades 3-4, with re-introduction of sunitinib at the same dose (grade 3 ) or at a reduced dose (grade 4 ) once diarrhea has improved to grade $\leq 1$. A dose-reduction may be preferred following grade 3 diarrhea on an individual patient basis.

o Nausea/vomiting: anti-emetic agents should be used early. Proton pump inhibitors will protect the gastric mucosa but should not be given within $2 \mathrm{~h}$ of sunitinib as they may interfere with its absorption and metabolism. Ondansetron and related drugs are not recommended since they may interfere with sunitinib metabolism through the CYP3A4 pathway. Dose adjustments of sunitinib are seldom necessary.

- Fatigue; exclude secondary cause(s); interruption of sunitinib is recommended for grade 3 fatigue, with re-introduction of sunitinib at a reduced dose once fatigue has improved to grade $\leq 1$. 
- Dermatological AEs rarely require dose reduction; interruption of sunitinib is recommended for grades 3-4, with re-introduction of sunitinib at a reduced dose once the AE (usually HFS) has improved to grade $\leq 1$.

- For oral toxicity, stomatitis, and mucositis symptomatic management is via dietary modification and oral care, dose adjustments / interruptions for oral toxicity are seldom necessary.

- Thyroid dysfunction: regular surveillance of thyroid function is recommended (initially 12-weekly and symptom-directed). Overt hypothyroidism should be treated with thyroid hormone replacement therapy according to local guidelines. Asymptomatic subclinical hypothyroidism should be monitored and treated if hypothyroidism becomes overt; sunitinib dose modifications are generally not required.

- Cardiovascular events: periodic monitoring (ECG and electrolytes) should be considered and additional monitoring for signs and symptoms of congestive heart failure (CHF) employed. The dose of sunitinib should be interrupted and/or reduced in patients without clinical evidence of CHF but with an ejection fraction $<50 \%$ and $>20 \%$ below baseline.

- Thromboembolic events have been treated successfully in other settings with low molecular weight heparin for 3-6 months without bleeding complications. Continued sunitinib is at the physician's discretion; interruption should be considered for patients in cardiorespiratory compromise with later reintroduction at the same dose level. Oral anti-vitamin K should be avoided as it interacts with both sunitinib and heparin. Asymptomatic pulmonary emboli should be treated on an individual patient basis, balancing potential risks and benefits.

In general, discontinuation of therapy is recommended for any grade 4 adverse events according to CTC [3], but there may be few exceptions, depending on the circumstance, in which clinical judgement can be used and it might be considered to restart therapy with dose reduction (see above).

\section{Monitoring of adverse events}

Patients should be reviewed every 4 weeks while on treatment; with long-term therapy ( $>2 \mathrm{yr})$ and good tolerability clinical follow-up can be reduced to 3-monthly.

Before starting sunitinib the following investigations should be done:

- Thorough physical investigation (including baseline BP and heart rate) and assessment of comorbidities (e.g. hypertension, history of bleeding, etc.).

- Laboratory: Blood cell count, blood glucose, electrolytes, calcium, serum phosphate, creatinine, transaminases and thyroid ECG (and estimation of left ventricular ejection fraction (LVEF) for patients with a cardiac history). 
During treatment the following investigations are recommended:

- Assessment of the general condition, PS and weight every 4 weeks.

- Laboratory parameters should be measured regularly after 2 weeks, and thereafter at least on a monthly basis (more frequently if needed) within the first 3 months: blood cell count, blood glucose, electrolytes, calcium, serum phosphate and creatinine; the frequency of these tests may be reduced thereafter depending on the stability of the patient.

- Thyroid functions tests 12-weekly.

- ECG and LVEF as clinically indicated.

\section{Evaluation of treatment effect}

Biochemical markers: CgA or NSE (if elevated prior to therapy); specific biomarkers (depending on functionality).

Tumor follow-up: Conventional imaging, either CT or MRI (whatever method depicts tumor lesions most accurately) every 3 months. With long-term therapy and stable disease the staging interval may be prolonged to 6-monthly. Although RECIST evaluation has limitations in NET response assessment, these criteria are still considered the reference method [41].

\section{Patient information}

The patients should:

- Be informed that hair may go grey, and that this reverses after stopping sunitinib.

- Be informed that a discoloration of the skin may occur and that dysgeusia is common.

- Be advised that blood pressure may increase and require treatment.

- Know that bleeding may occur, requiring immediate attention.

- Keep well hydrated, avoiding foods which may exacerbate diarrhea in individual patients (e.g. caffeine, lactose-containing foods, fatty or high-fiber foods and fruits (except pectin-containing fruit (e.g., apples and bananas). These will vary from patient to patient.

- Know that grapefruit juice and Hypericum perforatum should not be taken while on sunitinib treatment.

- Be encouraged to take care of their hands and feet from the onset of treatment, including the use of moisturizing and urea-based creams; avoid rubbing (e.g., ill-fitting shoes), manicure/pedicure, etc.

- Know that skin toxicity may be reduced by avoiding hot showers, reducing sun exposure, and wearing loose-fitting clothing and comfortable shoes.

\section{Drug interactions}

In accordance with prescribing information for sunitinib, patients should try to avoid taking strong CYP3A4 inhibitors (e.g. ketoconazole, ritonavir, itraconazole, erythromycin, clarithromycin, etc.) and 
should not drink grapefruit juice. If no alternative is available, a dose reduction of sunitinib should be considered where sunitinib is co-administered with these medications.

Similarly, patients should try to avoid taking CYP3A4 inducers (e.g. rifampicin, dexamethasone, phenytoin, carbamazepine, phenobarbital or herbal preparations containing St. John's Wort/Hypericum perforatum); if no alternative is available, a dose increase of sunitinib might be necessary if patients are receiving CYP3A4 inducers as these may decrease sunitinib concentrations.

\section{Pregnancy and lactation}

Studies in animals have shown fetal malformations with sunitinib; it should not be used during pregnancy or in men/women not using effective contraception.

Lactation: It is not known whether sunitinib or its primary active metabolite is excreted in human milk; however, because of the potential for serious adverse reactions in breast-feeding infants, women should not breast-feed while taking sunitinib.

\section{Antibes Consensus Conference participants}

Arnold, R. (Munich, Germany), Bartsch, D.K. (Department of Surgery, Philipps University, Marburg, Germany), Baudin, E. (Département de Médecine, Gustave Roussy, 114, rue Édouard-Vaillant, Paris South University, Villejuif Cedex 94805, France), Borbath, I. (Service de Gastroenterologie, Cliniques Universitaires St-Luc, Bruxelles, Belgium), Capdevila, J. (Vall d'Hebron University Hospital, Teknon Institute of Oncology, Barcelona, Spain), Chen, Y.-J. (Peking Union Medical College Hospital, Chinese Academy of Medical Sciences, Beijing, China), Couvelard, A. (Service de Pathologie, Hôpital Bichat, Paris, France), Cwikla, J.B. (Department of Radiology, Faculty of Medical Sciences, University of Warmia and Mazury, Olsztyn, Poland), Davies, P. (Neuroendocrine Tumour Unit, Royal Free Hospital, London, United Kingdom), Delle Fave, G. (Department of Digestive and Liver Disease, Ospedale Sant'Andrea, Rome, Italy), Ferone, D. (Department of Endocrine and Metabolic Sciences, University of Genoa, Genoa, Italy), Grozinsky-Glasberg S. (Neuroendocrine Tumor Unit, Endocrinology and Metabolism Service, Department of Medicine, Hadassah-Hebrew University Medical Center, Jerusalem, Israel), Gross, D. (Department of Endocrinology \& Metabolism, Hadassah University Hospital, Mevasseret Tsion, Israel), Grossman, A. (Oxford Centre for Diabetes, Endocrinology and Metabolism, Churchill Hospital, Oxford, United Kingdom), Hicks R.J. (Cancer Imaging, the Peter MacCallum Cancer Centre, Melbourne), Hörsch, D. (Gastroenterology and Endocrinology Center for Neuroendocrine Tumors Bad Berka, Bad Berka, Germany), Tiensuu Janson, E. (Deptartment of Endocrine Oncology, Uppsala University Hospital, Uppsala, Sweden), Jensen, R.T. (Digestive Diseases Branch, NIH, Bethesda, Md., USA), Kaltsas, G. (Department of Pathophysiology, Division of Endocrinology, National University of Athens, Athens, Greece), Knigge, U.

(Neuroendocrine Tumor Center of Excellence, Rigshospital, Copenhagen University Hospital, 
Copenhagen, Denmark), Kos-Kudla, B. (Department of Endocrinology, Medical University of Silesia, Katowice, Poland), Krenning, E.P., (Cyclotron Rotterdam BV, Erasmus MC, Rotterdam, The Netherlands), Kwekkeboom, D.J. (Department of Internal Medicine, Division of Nuclear Medicine, ENETS Centre of Excellence Rotterdam, Erasmus MC, Rotterdam, The Netherlands), Niederle, B. (Department of Surgery, Medical University of Vienna, Vienna, Austria), Nieveen van Dijkum, E.J.M. (Department of Surgery, Academic Medical Center, Amsterdam, the Netherlands), Öberg, K. (Department of Medical Sciences, Endocrine Oncology Unit, University Hospital, Uppsala, Sweden), O’Toole, D. (NET Centre, St. Vincent's University and Department of Clinical Medicine, St James Hospital and Trinity College, Dublin, Ireland), Pape, U.-F. (Department of Hepatology and Gastroenterology, Campus Virchow Klinikum, Charité Universitätsmedizin Berlin, Berlin, Germany), Pascher, A. (Department of Surgery, Charité-Universitaetsmedizin Berlin, Berlin, Germany), Perren A. (Institute of Pathology, University of Bern, Switzerland), Ramage, J. (Gastroenterology Department, Hampshire Hospitals NHS Trust, Hampshire, United Kingdom), Reed, N. (Beatson Oncology Centre, Gartnavel General Hospital, Glasgow, United Kingdom), Rindi, G. (Institute of Anatomic Pathology, Policlinico A. Gemelli, Università Cattolica del Sacro Cuore, Rome, Italy), Ruszniewski, P. (Department of Gastroenterology, Beaujon Hospital, Clichy, France), Scoazec, J.-Y. (Services de pathologie morphologique et moléculaire, Département de biologie et pathologie médicales, Gustave Roussy Cancer Campus, Villejuif, France), Sundin, A. ( Department of Radiology, Inst. Surgical Sciences, Uppsala University, Uppsala University Hospital, Uppsala, Sweden), Toumpanakis, C. (Neuroendocrine Tumour Unit, Royal Free Hospital, London, United Kingdom), Vullierme M.-P. (Service de Gastroentérologie,Hôpital Beaujon, Clichy, France), Welin, S. (Department of Medical Sciences, Endocrine Oncology, Uppsala University, Sweden), Wiedenmann, B. (Department of Hepatology and Gastroenterology, Campus Virchow Klinikum, Charité Universitätsmedizin Berlin, Berlin, Germany) 


\section{References}

1. Pavel M, O'Toole D, Costa F, Capdevila J, Gross D, Kianmanesh R, et al: Consensus Guidelines Update for the Management of Distant Metastatic Disease of Intestinal, Pancreatic, Bronchial Neuroendocrine Neoplasms (NEN) and NEN of Unknown Primary Site. Neuroendocrinology 2016;103:172-185.

2. Garcia-Carbonero R, et al: ENETS Consensus Guidelines for the Standards of Care in Neuroendocrine Neoplasms. Systemic Therapy: Chemotherapy. Neuroendocrinology 2017; $\mathrm{xxx}$

3. Common Terminology Criteria for Adverse Events (CTCAE) (v4.03: June 14, 2010). U.S. Department of Health and Human Services. National Institutes of Health. National Cancer Institute. http://evs.nci.nih.gov/ftp1/CTCAE/CTCAE_4.03_2010-0614_QuickReference_5x7.pdf

4. Rinke A, Krug S. Neuroendocrine tumours - Medical therapy: Biological. Best Pract Res Clin Endocrinol Metab 2016; 1:79-91.

5. Rinke A, Müller HH, Schade-Brittinger C, Klose KJ, Barth P, Wied M, et al: Placebocontrolled, double-blind, prospective, randomized study on the effect of octreotide LAR in the control of tumor growth in patients with metastatic neuroendocrine midgut tumors: a report from the PROMID Study Group. J Clin Oncol 2009; 28:4656-63.

6. Caplin ME, Pavel M, Ćwikła JB, Phan AT, Raderer M, Sedláčková E, et al: Lanreotide in metastatic enteropancreatic neuroendocrine tumors. N Engl J Med 2014; 371:224-233.

7. van Essen M, Sundin A, Krenning EP, Kwekkeboom DJ: Neuroendocrine tumours: the role of imaging for diagnosis and therapy. Nat Rev Endocrinol 2014;10:102-114.

8. Strosberg JR, Benson AB, Huynh L, Duh MS, Goldman J, Sahai V, et al: Clinical benefits of above-standard dose of octreotide LAR in patients with neuroendocrine tumors for control of carcinoid syndrome symptoms: a multicenter retrospective chart review study. Oncologist 2014; 19:930-936.

9. Kaltsas G et al: ENETS Consensus Guidelines for the Standards of Care in Neuroendocrine Tumors: Pre- and Perioperative Therapy in Patients with Neuroendocrine Tumors. Neuroendocrinology 2017; xxx

10. Strosberg J, El-Haddad G, Wolin E, Hendifar A, Yao J, Chasen B, et al: Phase 3 Trial of 177Lu-Dotatate for Midgut Neuroendocrine Tumors. N Engl J Med 2017; 376:125-135.

11. Bornschein J, Drozdov I, Malfertheiner P: Octreotide LAR: safety and tolerability issues. Expert Opin Drug Saf 2009; 8:755-768.

12. Lima-Martínez MM, López-Méndez G, Mangupli R: Bradicardia sinusal inducida por octreotide en un varón con acromegalia. Endocrinol Nutr 2013;60:e7-e9.

13. Dilger J, Rhoe E, Que F, Sprung J: Octreotide-Induced Bradycardia and Heart Block During Surgical Resection of a Carcinoid Tumor. Anesth Analg 2004; 98:318-320.

14. Herrington AM, George KW, Moulds CC: Octreotide-Induced Bradycardia. Pharmacotherapy 1998;18: 413-416.

15. Ogmen B, Polat B, Cuhaci N, Aydin C, Ersoy R, Cakir B: Lanreotide-induced bradycardia and supraventricular extrasystoles. Endocrine Abstracts 2014; 35: P916; DOI:10.1530/endoabs.35.P916

16. Haugen B: Drugs that suppress TSH or cause central hypothyroidism. Best Pract Res Clin Endocrinol Metab 2009; 23: 793-800.

17. Fiebrich HB, Van Den Berg G, Kema IP, Links TP, Kleibeuker JH, Van Beek AP, et al: Deficiencies in fat-soluble vitamins in long-term users of somatostatin analogue. Aliment Pharmacol Ther. 2010; 32:1398-1404. 
18. Saif MW, Larson H, Kaley K, Shaib W: Chronic octreotide therapy can induce pancreatic insufficiency: a common but under-recognized adverse effect. Expert Opin Drug Saf 2010; 9:867-873.

19. Wolin EM, Jarzab B, Eriksson B, Walter T, Toumpanakis C, Morse MA, et al: Phase III study of pasireotide long-acting release in patients with metastatic neuroendocrine tumors and carcinoid symptoms refractory to available somatostatin analogues. Drug Des Devel Ther 2015; 9:5075-5086.

20. Plöckinger U, Dienemann D, Quabbe HJ: Gastrointestinal side-effects of octreotide during long-term treatment of acromegaly. J Clin Endocrinol Metab 1990; 71:1658-1662.

21. Abell S, Teng J, Dowling A, Hofman MS, MacIsaac RJ, Sachithanandan N: Prolonged life-threatening hypoglycaemia following dose escalation of octreotide LAR in a patient with malignant polysecreting pancreatic neuroendocrine tumour. Endocrinol Diabetes Metab Case Rep 2015; 140097. doi: 10.1530/EDM-14-0097.

22. Knigge U et al: ENETS Consensus Recommendations for the Standards of Care in Neuroendocrine Neoplasms: Follow-Up and Documentation, Neuroendocrinology 2017; xxx

23. Drug Development and Drug Interactions: Table of Substrates, Inhibitors and Inducers. U.S. Food and Drug Administration (FDA).

http://www.fda.gov/Drugs/DevelopmentApprovalProcess/DevelopmentResources/DrugInterac tionsLabeling/ucm093664.htm

24. de Menis E, Billeci D, Marton E, Gussoni G: Uneventful pregnancy in an acromegalic patient treated with slow-release lanreotide: a case report. J Clin Endocrinol Metab 1999; 84:1489.

25. Briggs G, Freeman R, Yaffe S: Octreotide- Pregnancy Summary. In: Drugs in Pregnancy and Lactation: A Reference Guide to Fetal and Neonatal Risk. Lippincott Williams \& Wilkins, 2011

26. Arnold R, Rinke A, Klose KJ, Müller HH, Wied M, Zamzow K, et al: Octreotide versus octreotide plus interferon-alpha in endocrine gastroenteropancreatic tumors: a randomized trial. Clin Gastroenterol Hepatol 2005;3:761-771.

27. Faiss S, Pape UF, Böhmig M, Dörffel Y, Mansmann U, Golder W, et al: International Lanreotide and Interferon Alfa Study Group. Prospective, randomized, multicenter trial on the antiproliferative effect of lanreotide, interferon alfa, and their combination for therapy of metastatic neuroendocrine gastroenteropancreatic tumors-the International Lanreotide and Interferon Alfa Study Group.J Clin Oncol 2003;21:2689-2696.

28. Oberg K: Interferon in the management of neuroendocrine GEP-tumors: a review.Digestion. 2000;62 (Suppl 1):92-97.

29. Yao J, Guthrie K, Moran C, Strosberg J, Kulke M, Chan J et al: SWOG S0518: Phase III prospective randomized comparison of depot octreotide plus interferon alpha-2b versus depot octreotide plus bevacizumab (NSC \#704865) in advanced, poor prognosis carcinoid patients (NCT00569127). J Clin Oncol 2015; 33: 4004.

30. Yao JC, Phan A, Hoff PM, Chen HX, Charnsangavej C, Yeung SC, et al:Targeting vascular endothelial growth factor in advanced carcinoid tumor: a random assignment phase II study of depot octreotide with bevacizumab and pegylated interferon alpha-2b. J Clin Oncol 2008; 26:1316-1323.

31. Pavel ME, Baum U, Hahn EG, Schuppan D, Lohmann T: Efficacy and tolerability of pegylated IFN-alpha in patients with neuroendocrine gastroenteropancreatic carcinomas. J Interferon Cytokine Res 2006; 26:8-13.

32. Hiratsuka M1, Minakami H, Koshizuka S, Sato I: Administration of interferon-alpha during pregnancy: effects on fetus. J Perinat Med 2000;28:372-376. 
33. Yao JC, Shah MH, Ito T, Bohas CL, Wolin EM, Van Cutsem E, et al: RAD001 in Advanced Neuroendocrine Tumors, Third Trial (RADIANT-3) Study Group. Everolimus for advanced pancreatic neuroendocrine tumors. N Engl J Med 2011;364:514-523.

34. Yao JC, Fazio N, Singh S, Buzzoni R, Carnaghi C, Wolin E, et al: Everolimus for the treatment of advanced, non-functional neuroendocrine tumours of the lung or gastrointestinal tract (RADIANT-4): a randomised, placebo-controlled, phase 3 study. Lancet 2016;387:968977.

35. Kulke MH, Bergsland EK, Yao JC: Glycemic control in patients with insulinoma treated with everolimus. N Engl J Med 2009;360:195-197.

36. Pavel ME, Hainsworth JD, Baudin E, Peeters M, Hörsch D, Winkler RE, et al: Everolimus plus octreotide long-acting repeatable for the treatment of advanced neuroendocrine tumours associated with carcinoid syndrome (RADIANT-2): a randomised, placebo-controlled, phase 3 study. Lancet 2011; 378: 2005-2012.

37. Trelinska J, Dachowska I, Kotulska K, Fendler W, Jozwiak S, Mlynarski W: Complications of mammalian target of rapamycin inhibitor anticancer treatment among patients with tuberous sclerosis complex are common and occasionally life-threatening. Anticancer Drugs 2015; 26:437-442.

38. Grünwald V, Weikert S, Pavel ME, Hörsch D, Lüftner D, Janni W, et al: Practical management of everolimus-related toxicities in patients with advanced solid tumors. Onkologie 2013; 36:295-302.

39. Rugo HS, Seneviratne L, Beck JT, Glaspy JA, Peguero JA, Pluard TJ et al: Prevention of everolimus/exemestane (EVE/EXE) stomatitis in postmenopausal (PM) women with hormone receptor-positive $(\mathrm{HR}+)$ metastatic breast cancer $(\mathrm{MBC})$ using a dexamethasone-based mouthwash (MW): Results of the SWISH trial. J Clin Oncol 2016; 34(suppl): abstract 525.

40. Catapano AL, Graham I, De Backer G, Wiklund O, Chapman MJ, Drexel H, et al: 2016 ESC/EAS Guidelines for the Management of Dyslipidaemias: The Task Force for the Management of Dyslipidaemias of the European Society of Cardiology (ESC) and European Atherosclerosis Society (EAS) Developed with the special contribution of the European Assocciation for Cardiovascular Prevention \& Rehabilitation (EACPR). Eur Heart J 2016 ;Aug 27. pii: ehw272. [Epub ahead of print]

41. Sundin et al: ENETS Consensus Guidelines for the Standards of Care in Neuroendocrine Tumors: Radiological, Nuclear Medicine \& Hybrid Imaging. Neuroendocrinology 2017; xxx

42. Margoles HR, Gomez-Lobo V, Veis JH, Sherman MJ, Moore J Jr: Successful maternal and fetal outcome in a kidney transplant patient with everolimus exposure throughout pregnancy: a case report. Transplant Proc 2014; 46:281-283.

43. Raymond E, Dahan L, Raoul JL, Bang YJ, Borbath I, Lombard-Bohas C, et al: Sunitinib malate for the treatment of pancreatic neuroendocrine tumors. N Engl J Med 2011;;364:501513.

44. Valle JW, Faivre S, Hubner RA, Grande E, Raymond E: Practical management of sunitinib toxicities in the treatment of pancreatic neuroendocrine tumors. Cancer Treat Rev 2014; 40:1230-1238.

45. Kollmannsberger C, Bjarnason G, Burnett P, Creel P, Davis M, Dawson N et al: Sunitinib in metastatic renal cell carcinoma: recommendations for management of noncardiovascular toxicities. Oncologist 2011;16:543-553.

46. Faivre S, Delbaldo C, Vera K, Robert C, Lozahic S, Lassau N et al. Safety, pharmacokinetic, and antitumor activity of SU11248, a novel oral multitarget tyrosine kinase inhibitor, in patients with cancer. J Clin Oncol 2006; 24:25-35. 
47. Funakoshi T, Shimada YJ: Risk of hypothyroidism in patients with cancer treated with sunitinib: a systematic review and meta-analysis. Acta Oncol 2013;52:691-702.

48. NCCN Clinical Practice Guidelines in Oncology (NCCN Guidelines $\left.{ }^{\circledR}\right)$ : Myeloid Growth Factors, Version 2.2016.

https://www.nccn.org/professionals/physician_gls/recently_updated.asp

Neuroendocrinology (International Journal for Basic and Clinical Studies on Neuroendocrine Relationships) Journal Editor: Millar R.P. (Edinburgh) ISSN: 0028-3835 (Print), elSSN: 1423-0194 (Online)

www.karger.com/NEN

Disclaimer: Accepted, unedited article not yet assigned to an issue. The statements, opinions and data contained in this publication are solely those of the individual authors and contributors and not of the publisher and the editor(s). The publisher and the editor(s) disclaim responsibility for any injury to persons or property resulting from any ideas, methods, instructions or products referred to in the content. Copyright: All rights reserved. No part of this publication may be translated into other languages, reproduced or utilized in any form or by any means, electronic or mechanical, including photocopying, recording, microcopying, or by any information storage and retrieval system, without permission in writing from the publisher or, in the case of photocopying, direct payment of a specified fee to the Copyright Clearance Center. 